\title{
Hermann Cohens systematische Arbeit im Dienste des kritischen Idealismus.
}

Von Albert Görland.

1871 erschien „Kants Theorie der Erfahrung". Mit diesem Buche erschloss Cohen den Weg des tiefen, zukunftkrậftigen Kantverständnisses; und zugleich für sich den Weg auf ein deutsches Katheder für Philosophie. Das war damals. Wir müssen heute mit tieferem Ernste denn je Friedrich Albert Lange danken, dass er die edle Bereitschaft zeigte, für ein Werk sich einzusetzen, das ihn selbst zum Umlernen zwang; und nicht etwa nur zum Zwecke einer wirkliç buchstabengetreuen, sogenannt philologischen Kantkenntnis, sondern bezüglich der zentralen Gedanken kruitischen Philosophierens. Denñ es giñg von diesem Bùche der Geist einer nèuèn Orientierung aus über das, was Philosophie bedeutèn müsse. Schon hier werden bestimmt und klar die Bemühungen um die Geschichte der Philosophie für die Zwecke der systematischen Philosophie in Anspruch genommen. Und zwar ist von Anfạng an das historische Interesse bei Cohen tiefër begründet als bloss darin, illustrierende Beispiele für die ëigene Entwicklung systematischer Motive bereitstellen zu können. So sagt er in seinem ersten und zugleich bestimmenden systematischen Buche: „Das Prinzip der Infinitesimal-Methode und seine Geschichte" (1883): "Nirgend ist es mir so sehr Bedürfnis gewesen und nirgend auch so uñmittelbar nützlich erschienen, zugleich mit der Durchführung eines systematisch entscheidenden Gedankens seine geschichtliche Entwicklung zu verfolgen". Er beabsichtigte hier, den Begriff der Realität in seinem Werte für die Begründung der Erkenntnis auszuzeichnen. Und also muśste ein Prinzip der Mathematik in philosophische Diskussion gẹnommen werden. Da stellte sich die Befürchtung ein, als müsse der Philosoph einen Eingriff in das Detail wissenschaftlicher Forschung begehen. Betrachtet der Philosoph aber den Ursprung 
des Gedankens im geschichtlichen Zusammenhange, so ist er davor geschützt. „Was sonach die Kategorie der Realität für den Begriff der Materie und Natur, wie für das Problem des Bewusstseins zu bedeuten und zu leisten habe, sollen wir bei denen erfragen, deren ineinander greifende Arbeit die neue Wissenschaft entdeckt hat. Galilei, Kepler und Newton, Descartes und Leibniz mit ihren Genossen und Vermittlern können uns Kant begreifen lehren und in seinem Geiste das Werk der Philosophie fortzuführen helfen."

In dieser Doppelrichtung des Interesses von historischer Orientierung auf systematische Entwicklung und umgekehrt liegt der trotz aller Einzelwandlungen des Cohenschen Denkens von Anfang an einheitliche Charakter seiner Weltanschauung und die Tiefe seiner Wirkung als Lehrer. "Man weiss, dass ich die Kraft der Vernunft nicht abgetrennt zu denken vermag von ihrer geschichtlichen Kontinuität". Am Schlusse seiner "Logik der reinen Erkenntnis" prägt er für diesen geschichtlichen Sinn des Idealismus den Ausdruck des klassischen Idealismus.

Es muss erkannt werden, dass dieses unabgesehlossene Durchdringen des geschichtlichen Materials mit dem systematischen Interesse der wahren historischen Objektivität nur dient, wofern ihr die Aufgabe obliegt, Geschichte, d. h. Kontinuität der Zeiten auf Grund ihrer Problemarbeit darzustellen, das historische Material als einen Schatz ewiger Rrobleme lebendig zu halten. Und ebenso muss erkannt werden, dass die unabgebrochen mögliche Kontrolle und Rechtfertigung vor der Geschichte dem Vorwärtsschreiten des Systematikers diejenige Freiheit der Wandlung und Zuversicht des Problementwurfs ${ }^{\complement}$ gewährt, die das Temperament des ' wissenschaftlichen Versuches ist im Widerspruch zum blinden Eigensinn einer auf sich beschränkten Spekulation. So können und werden tiefgreifende Wandlungen im Urteil des Systematikers darüber getrost eintreten, in welcher Weise die Einzelmotive zu einander stehen nach ihrem Anteil am systematischen Aufbau, und jene Wandlungen innerhalb sicherer methodischer Grenzen eintreten.

So liegen auch bei Cohen tiefgreifende Wandlungen des Denkens vor, die sich von der "Infinitesimalmethode" (1883) bis zur "Logik der reinen Erkenutnis" (1902) vollziehen und sich schon im Titel beider Bücher ausprägen; denn die erstere Schrift führt sich im Nebentitel als „Ein Kapitel zur Grundlegung der Erkenntniskritik " ein, wovon das zweite Werk nichts mehr weiss. 1883 stand für Cohen einer Erkenntniskritik noch als 
Disziplin eine Logik vorauf. Die einleitende Erörterung bemühte sich, zwischen beiden einen scharfen Unterschied beranszustellen; dazn nötigte der Infinitesimalbegriff. Denn seine Definition war bedingt durch die Festsetzung der Grenzen von Anschauung und Denken, der durch sie bezeichneten Methoden. Nun gehört aber die Unterscheidung von Denken und Anschauung nicht der Logik an, sondern lediglich die von der Anschauung abgetrennten Denkverhältnisse; um so weniger gehört der Begriff vor ihr Forum, dessen Schwierigkeit in dem Grenzstreit beider liegt.

Somit handelte es sich um zwei Arten von Erkenntnismitteln des Bewusstseins: Anschauung und.Denken. Hat die Logik (die "traditionelle Logik") die Sicherung des Erkennens von Seiten der Denkgrundlagen zu leisten, so muss es eine Disziplin geben, die alle die andern Mittel des Bewusstsein zu enthalten hat, die die gegenständliche Erkenntnis erzeugen und gewährleisten. Zu diesen andern Mitteln gehört die Anschauung, die der Logik fremd ist, sich aber mit den Deñkmitteḷn zúm Zwecke der Erkenntnis verbinden muss. Wo es sich nun ùm die Verbindung dieser beiden Mittel, wo es sich um Erkenntnis handelt, da tritt die Untersuchung der Erkenntniskritik ein. Somit steht Cohen 1883 der Kantischen Unterscheidung der Erkenntnismittel unmittelbar nahe. Und Erkenntniskiritik ist ihm gleichbedeutend mit der transzendentalen Logik. Ebenso bekennt sich Cohen zur Kantischen Rangordnung der Erkenntnisbedingungen, die der Zeit und dem Raum den ersten Platz unter ihnen anweist. „Mir liegt es fern, diesër Ansicht entgegentreten zu wollen".

Die "Logik" von 1902 ist "Log̣ik der reinen Erkenntnis". Sie lehnt den Unterschied von Logik und Erkenntniskritik ab. „Erkenntnistheorie ist ein unklarer Titel. Auch Erkenntniskritik kann nicht stichhalten." Dieser Gedanke schafft sich seinen scharfen Ausdruck im Titel dieser "Logik". Sie wird von der Untersuchung des ihr nun immanenten Begriffs "Erkenntriis" eingeleitet. Das "Denken" im Sinne der formalen Logik wird eingeschränkt auf einen ersten "Gesichtspunkt" und führt zur ersten der vier Urteilsgruppen, zu den Urteilen der Denkgesetze. Das "Denken" aber, das sich über die ganze "Logik der reinen Erikenntnis" ausbreitet, ist das Denken der Erkenntnis, dem nicht eine „Anschauung" noch zur Seite steht, hat also die Kompetenz des gegenständlichen Denkens der "transzendentalen Logik"; aber mit dem ausschlaggebenden Unterschied, dass ihr nicht mehr die 
„transzendentale Ästhetik“ in irgend einer „Rangordnung der Erkenntuisbedingungen" zugeordnet ist.

Diese überaus folgenreiche Trennung von der Kantischen Systematik musste vollzogen werden, wenn die fundamentale Entdeckung, dass das gewaltige Mittel der Gegenstandserkenntnis, das Infinitesimale, bislang nicht als solches der Philosophie zum Bewusstsein gekommen war, zu voller und reiner Wirkung gelangen sollte.

Wie natürlich sind auch schon in der Schrift von 1883 Spuren zu entdecken, dass dem Differentialbegriff als dem Begriff der intensiven Grösse oder der Realität der erste Platz gegenüber der sinnlichen Gegebenheit eingeräumt werden müsse (S. 128). Denn muss die kontinuierliche Einheit der intensiven Grösse als Ursprung gedacht werden, muss also das Unendliche aus sich das Endliche erzeugen (S. 32), so muss das Bedürfnis dringend empfunden werden, die Korrelation von Sinnlichkeit und Denken zu ändern und den Aufbau derjenigen Elemente, die die Erkenntnisse von den Gegenständen der mathematischen Naturwissenschaft bedingen, "allenfalls auch mit dem Denkmittel der Realität" zu beginnen (S. 130). Aber der Gedanke ist systematisch noch gefesselt durch die historische Rücksicht auf die "allgemein rezipierte Lehre Kants“: Nicht um ein schlechthin Erstes „Beginnen" braucht es sich zu handeln, sondern nur um ein Beginnen bezüglich der mathematischen Naturwissensehaft; dem wird dadurch genügt, dass die Realität sich schon in der Räumlichkeit durchsetzt, soweit sie die Geometrie konstituiert. Es bleibt bestehen, dass die "Kategorie" "der Realität in einem "Grundsatz", dem der intensiven Grösse oder der Antizipation, mit der "Anschaung" sich vereinbaren muss.

Die "Logik der reinen Erkenntnis" hebt mit scharfen Angriffen gegen diese- wichtigsten Pfeiler des Kantischen Systemes an. Gleichwohl hält Cohen den Sinn und Inhalt seiner Bücher über Kant im Ganzen aufrecht. „Beides schliesst sich nicht nur nicht aus und verträgt sich nicht nur zufällig in mir, sondern es ergänzt sich zur Einheit einer systematischen Arbeit." Denn die Polemik gegen die Systematik Kants geschieht in der vollkommenten Einheit mit Kant gemäss seiner unvergänglichen methodischen Anweisung der Philosophie auf das Faktum der Wissenschaft. Durch dieses Verweisen der Philosophie anf die Prinzipien und Grundsätze der mathematischen Natur- 
wissenschaft konstituiert Kant die Wissenschaft der Philosophie, im Sinne der Kritik. Und diese Entdeckung der Kritik und deren Ausgestaltung bedeutet den bleibenden weltgeschichtlichen Wert der Kantischen Gedankenwelt, wie sehr auch aller noch so wichtige Einzelinhalt der Kantischen Gedanken wieder mag in Fluss gebracht werden müssen.

Unermüdet ist von Seiten Cohens und nach ihm der Marburger Schule dies Wort als Weckruf hinausgetragen: dass Philosophie als Wissenschaft allererst möglich wird, wenn sie ihr Problem aus der Tatsache der Prinzipien der Wissenschaft schöpft und sich nicht in irgend einer unmittelbaren Arbeit am Problem der Empfindung versucht. Aus zwiefachem Grunde wollen wir heute erneut auf diesen Gedanken Cohens, durch den er uns Kant ganz eigentlich erst entdeckt hat, aufmerksam machen.

Zunächst deshalb, weil immer von neuem die Bemühung auftaucht; in. Cohen und seiner Schule Fichtesche oder Hegelsche Tendenzen zu wittern. Demgegenüber ist zì sagen, dass diese Schule nicht das geringste Interesse aufbringen kann, sich einen "Standpunkt" zu markieren aus Distanzbestimmungen zu vorhandenen philosophischen "Syștemen"; und also wehrt sie sich nicht dagegen, noch sucht sie dạn Schutz, dass etwelcher Gleichklang von Worteñ oder Motivien ża diesem oder jenem philosophischen Systeme gefünden werden kann. Lehnt also die "Logik“ es ab, eine Lehre von der Șinnlichkeit dem Denken voraufzuschicken; fängt sie also mit dem Denken an, das in sich selbst und ausschliesslich reine Errkęnntnis, das heisst die Gegenständlichkeit der wissensçhaftlichen Erfahrung zur Erzeugung zu bringen habe; bekennt sie sich zur Parmenideischen Identität von Denken und Sein - so ist für diese (wie man sagt) abgründlichen und wildẹn Spekulationen der Gerichtshof nicht die Geschichte der philosophischen Systeme, sơndern die Geschichte der mathematíschen Naturwissensehaft. In ihr liegt die Gesetzesfessel des kritischen Idealismus, aber auch seine Zuversicht, dass er bestehen werde im Urteilsspruch der Zeiten.

Añdererseits aber auch deshalb: Cohen hat seinen Namen in dez Geschichte der Philosophie am sichersten dadurch begründet, dass er dieses "Eins und Alles" des kritischen Philosophierens auch da zur Geltung gebracht hat, wo Kant diesem Grundgedanken nicht hat entsprochen oder entsprechen können. So beim Infinitesimalbegriff, wie wir sehon andeuteten; und der Ethik hat Cohen 
Hermann Cohens systematische Arbeit i. Dienste d. krit. Idealismus. $227^{\circ}$

ganz eigentlich erst diejenige methodische Grundlage gegeben, die der kantischen Forderung des "Transzendentalen" entspricht; die aber von Kant schon darum verfehlt ist, weil or von der Tugendlehre die Rechtslehre abgetrennt hat. Cohen aber führte den Kantischen Urgedanken, dass die Philosophie auf die Tatsache von Wissenschaften restringiert ist, auch für die Ethik durch und bezeichnete ihr in der Rechts- und Staatslehre diejenige Wissenschaft, in deren Tatsache für jede als Wissenschaft mögliche Ethik das Problem gesetzt ist. Damit ist der kritische Gedanke in bedeutsamster Weise gereinigt und weitergeführt worden.

Will man also von irgend einer Seite her zu einem Urteile darüber gelangen, was das Lebenswerk Cohens für die Philosophie bedeuten wird, so kann es nur vermöge der methodischen Gesinnung geschehen, zu der uns dieser universale Grundgedanke des kritischen Idealismus Recht und Kraft gibt.

Dieser Begriff des kritischen Idealismus als der Philosophie, sofern sie Wisisenschaft sein kann, stand Cohen von Anfang an taghell vor Augen, so auch in der "Infinitesimalmethode", deren besonders glückliche Formulierungen wir zitieren wollen:

Die Kritik entdeckt das Reine in der Vernunft, insofern sie die Bedingungen der Gewissheit entdeckt, auf dem die Erkenntnis als Wissenschaft beruht. Der kritische Idealismus hat also nicht sowohl Dinge und Vorgänge, auch nicht solche des Bewusstseins schlechthin, sondern wissenschaftliche Tatsachen zu seinen Objekten. Er zerlegt die Einzelwissenschaften auf die Voraussetzungen und Grundlagen hin, die in ihren Gesetzen und für sie angenommen werden. Was also die Wissensehaft zur Wissenschaft macht, welche Bedingungen ihrer Gewissheit sie voraussetzt, von welchen Grundsätzen ihre Wirklichkeit nach ihrem angenommenen Werte als Wissenschaft, (der also nicht etwa erst unter anmassliche Frage gestellt wird), ermöglicht wird: das ist die methodische Frage der in Kant reif gewordenen Philosophie. Die Differentia specifica des kritischen und des dogmatischen Idealismus liegt deutlichst und ausschliesslich in diesem Hinweis auf die Wissenschaft, in welcher allein Dinge gegeben und für die philosophischen Fragen angreifbar vorhanden sind. Alse; hat demnach die Philosophie keinerlei unmittelbaren Bezug auf die Eupfindung. Und also liegt das "erkennende Bewusstsein" als ein erforschbares Faktum nicht etwa in den psychologischen Ansichten von unserer sogenannten physio-psychischen Organisation. 
vor, sondern das "erkennende Bewusstsein" hat nur in den Tatsachen der wissenschaftlichen Erkenntnis diejenige Wirklichkeit, auf welche eine philosophische Untersuchung sich beziehen kann und nur beziehen darf, wenn sie den Gegenstand der Natur aus dem - Denken abzuleiten sich erkühnen will.

Man sollte hoffen können, dass eine solche Methode des Philosophierens und solcher Leitgedanke zur Systematik hinlänglich klar ist, auf dass der heillose Eklektizismus unserer Tage in eine Heerstrasse gemeinsamer Arbeit za leiten sein möchte.

Gehen wir näher hinan an den zentralen Begriff der "Logik", die als Logik der Erkenntnis mit dem Denken anfängt. selbst."

„Das Denken darf keinen Ürsprung haben ausserhalb seiner

Damit löst sich die „Logik" total von der Ökonomie Kantischer Begriffe. Denn das war der charakteristische Ausgáng bèim Aufbau des Kantischen Gebändes, dass das „Mannigfaltige der Anschauung“ einen solchen Ursprung der Eirkenntnis ausserhalb des Denkens bezeichnet. 'Das "Mannigfaltige" ist durch das Denken nicht zu schaffen, also auch nicht zu verantworten. Es ist ihm "gegeben“. Das ist eîn Fehler nicht allein in der Kantischen Terminologie, sondern in der gešanten Disposition. Denn die ureigne Selbständigkeit des Denkens wird dadurch beeinträchtigt.

Der wichtigste Fehler Kants, der den eben beregten erst möglich gemacht hat, war der, dass der Begriff der Zahl nur in èinerlei Gestalt, nur als konstante Zahl erschiēn, nicht auch und vor allem als Infinitesimalzahl. Und also liegt der Hauptmangel, der bestimmend auf die gesamte Disposition wirkte, dạin, dass Kant in dem Zurückgehen auf die einzelnen Prinzipien der Mathematik vor der neuen Mathematik halt machte; alšo handelt es sich um einen schlichten Mangel in der klaren Durchführung der ureignen Methode der Kritik. Gewiss ist der Grundbegriff der neuen Grössenlehre nach seiner Bedeutung für die "Realität" von ihm erkannt worden; aber er wurde nicht der Hébel der Kritik. Und diese Bedeutung kommt inm zu.

Auch nach Kant ist das neue Problem, das der Logik durch die neue. Mathematik und Naturwissenschaft gestellt ist, noch immer nicht erfasst. Die Logik hat damit bislang ihre eigentliche Aufgabe verfehlt. Denn sie muss Logik der mathematischen Naturwiissensehaft sein und darum vorzugsweise die Logik des Prinzips der Infinitesimalrechnung. Dieses Prinzip aber gewährt die unge- 
schmälerte Sicherung, die uneingeschränkte schöpferische Selbstständigkeit des reinen Denkens. Aus der Analysis des Unendlichen erst lässt sich die unersetzliche Bedeutung des Denkens als Erzeugung gewinnen; denn das Unendliche ist das Problem des Ursprungs.

Und demgemäss ist die „Logik der reinen Erkenntnis": "Logik des Ursprungs." "Das ist nicht nur die neue Gestalt, die wir der Logik zu geben versuchen, sondern auch ein neues Fundament, das in der neuen Wissenschaft in anerkanntem Gebrauche ist, welches jedoch in der bisherigen Logik als Fundament nicht erkannt ist."

Weil nun alle reinen Erkenntnisse Abwandlungen des Prinzips des Ursprungs sind, dieses also das Wesen der Logik bestimmt, so wollen wir es näher betrachten, und auch seine vollere Gestalt im Urteil der Realität, dem eigentlichen logischen Ort des Infinitesimalbegriffs.

Jede Wissenschaft konstituiert sich in Prinzipien, Axiomen, Definitionen; also Setzungen, die jedem anderen Inhalte der Wissenschaft gegenüber als Voraussetzung, als Ursprung und Quelle seiner Sicherheit dienen. Woher diese Voraussetzungen, diese Setzungen, denen jegliche Sicherheit alles späteren Inhalts der Wissenschaft verdankt werden muss? Gibt es z. B. eine streng notwendige und allgemeingültige Mathematik, so ist dieser Geltungswert durch die mathematischen Prinzipien gegeben; woher sind sie gegeben? Fängt mit ihnen alle Sicherung und alle Geltung der abgeleiteten Erkenntnisse an, so ist damit eine Schranke der Rechtfertigung der Wissenschaft über ihr Fundament gesetzt; denn mit solchen Anfängen der Sicherung hört die Sicherheit der Wissenschaft vielmehr zugleich auf.

Das Parallelenaxiom ist eine Voraussetzung der euklidisehen Geometrie; woher dieses? Wie rechtfertigt es sich als Fundament der Geltung der Mathematik? Etwa durch „Erfahrung“? Vielmehr ist die Erfahrung als die Einheit der Erfahrung gesichert erst durch die Sicherheit des Parallelenaxioms oder ein dem Ähnliches; sie soll sich in der unendlichen Arbeit der Wissenschaft primär aus dem Fundamente der Mathematik sichern; nicht umgekehrt.

So muss denn gefordert werden, dass die Frage auf das Fundament und seine Zulänglichkeit zurïck möglich ist; und dass die Antwort nicht "gegeben", sondern in der methodischen Einheit mit der Fragestellung gesucht werden muss, das heisst als 
Antwort dieses selben fragenden Denkens. Ist also z. B. das Parallelenaxiom eine Voraussetzung des mathematischen Denkens, so muss gefordert werden, dass es seine Rechtfertigung als solche Voraussetzung durch eben dieses mathematische Denken zu erlangen habe.

Wie kann aber das mathematische Denken hinter seine Voraussetzung zurüokfragen? Liegt nicht hinter oder vor der Voraussetzung methodisch das - Nichts? So offenbart sieh in dieser ganzen Fragestellung ein Notstand der Wissenschaft. Die Wissenschaft schwebt so lange im Leeren, so lange hinter diese Voraussetzung, hinter das „Prinzip“. nicht zurückgefragt ist.

Dieses - Nichts kann zwar nicht die Antwort der Rechtfertigung sein; es ist vielmehr die schwerstwiegende Mahnung zur Rechtfertigung; die eindringlichste Warnung vor dem "Anfang“. Gleichwohl aber muss die Wissenschaft so vor das "Erste", das Prinzip, die Voraussetz̃ung zurückgehen, als ginge sie in das Nichts. Damit zeigt sich das Nichts als ein Operationsbegriff der Wissenschaft, insofern es das Richtnngszeichen der Rechtfertigung der Voraússetzung ist, die nicht durch den Erfolg geschieht, sondern durch eine nächște Vंoraussetzung dieser Voraussetzung.

Welche ist die nächste? Das ist die bestimmtere Frage, die von der Methode der Rechtfertigung gestellt wird. Es ist der kontinuierlich nächste Zurüekschritt vor die Voraussetzung in der Richtung auf das Nichts zu tun.

So erlangte das Parallelenaxiom als Prinzip der (von nun an eũklidisch genannten) Geometrie seine Rechtfertigung durch den prinzipiellen Entwurf der Metageometrie; in ihr hat es seine Rechtfertigung und Sicherung erfahren, indem es als ein Spezialfall in allgemeinerer und tieferer Gesetzlichkeit begründet worden ist. Und dieser Weg von der euklidischen Geometrie zurück in der Richtung auf das Nichts zur uneaklidischen Geometrie vollzog sich in strengster Kontinuität des Rückganges' von der euklidischen Geometrie her.

In vollkommen entsprechender Methodik gelangt die neueste Physik zu den Begriffen der "wahren Bewegung" und der „richtigen Zeit". (cf. Föppl, Techn. Mechan. Bd. VI.)

Somit ist die Kontinuität das Gesetz der Operation, das vom wissenschaftlichen Denken in der Rechtfertigung über sein Fundament befolgt wird; durch die Kontinuität der Begründung der 
Hermann Cohens systematische Arbeit i. Dienste d. krit. Idealismus. 231

Voraussetzung sichert sich das Denken vor dem Unbegriffe des Nichts, auf das hin gleichwohl das Gesetz der Operationen sich richtet.

Diese Forderung, dass dem Denken (der Wissenschaft) keine Voraussetzung gegeben sein darf, dass es aus keinem Anfange sich erheben darf, als sofern es ihn innerhalb seiner Methode selbst beglaubigen kann: diese Forderung heisst das Denkgesetz des Ursprungs.

Nun gibt es für keine Wissenschaft ein absolut erstes „Prinzip“; denn das Gesetz der wissenschaftlichen Operation: die Kontinuität des Rückganges hinter das Prinzip zurück hält die Begründung des jetzt noch "ersten Anfanges" unbegrenzt vom Nichts ab. Also reisst die Forderung der Begründung nirgends ab; die Forderung, dass das Denken sich seinen "Anfang“ in einem prinzipiell früheren zu erzeugen habe, išt unbegrenzt. Solches besagt das Denkgesetz des Ursprungs.

Die Forderung des Ursprungs ist somit die Forderung einer unendlichen methodischen Gerechtsame des Denkens. Jede Rechtfertigung eines Anfanges (Prinzips) auf seinen methodischen Ursprung hin macht diesen Ursprung zu einem Anfang, vor dem nur die Forderung, die das Denkgesetz des Ursprungs ausspricht, (dass das Denken seinen Inhalt zu erzeugen, sich in methodischer Einheit unbegrenzt zu rechtfertigen hat), von neuem sich erhebt; und so ins Unendliche weiter. -

Wir übergehén das Denkgesetz der Identität und des Wịderspruchs und treten in die zweite Klasse von Urteilen, denen der Mathematik ein. Hier muss vor allem die Forderung des Ursprungs erfüllt werden. Ja, hätte nicht in der infinitesimalen Zahl der Mathematik das klassische Beispiel des Ursprungs vorgelegen, so hätte dem Denkgesetz des Ürsprungs gleichsam das methodische Rückgrat gefehlt, sagt Cohen.

Bei der Darstellung dieses ersten der drei „Urteile der Mathematik" zeigt sich, wie kräftig der „Logik der reinen Erkenntnis" durch die "Infinitesimalmethode“ von 1883 vorgearbeitet worden ist und wie treu der kritischen Methode Cohens philosophisches Denken durch das Problemgebiet der neuen Wissenschaft bestimmt ist.

Leibniz nennt das Differential unendlich klein, weist aber allem Endlichen, soweit es in den Bereich der Mathematik fällt in dieser neuen Zahl seinen zulänglichen Grund an. Das dx ist 
der Ursprung des $x$, mit dem die Analysis rechnet und das der Repräsentant des Endlichen ist. Ferner ergibt die Orientierung an Leibniz, dass das Infinitesimale nicht aus der Ausdehnung resultiert, sondern umgekehrt: es geht als deren Ursprung der Ausdehnung vorauf; „imo extensione prius". Also ist es im reinen Denken allein gegründet und bildet daraus den Grund des Endlichen. Demnach wird das Endliche begründet durch das Denken, das im Urteil des Unendlichkleinen sich vollzieht, and nicht durch Anschauung, nicht durch Empfindung.

Es bedarf kaum einer besonderen Frage, warum Cohen das Infinitesimale als die Realität bestimmt hat. Gleichwohl wollen wir ein paar Worte sagen, um die Realität davor zu bewahren, mit der "Wirklichkeit" verwechselt zu werden.

So wenig "Wirklichkeit" mit Wahrheit einerlei ist, so wenig auch mit der Realität. Denn Wirklichkeit ist die Charakteristik der Empfindung; Empfindung aber ist das Totalproblem für alle wissenschaftliche Arbeit und also die "Wirklichkeit" nicht ein methodisches İnstrument der Wissensçhaft; ein solehes aber stellt sich in der Analysis des Unendlichkleinen dar. Die Empfindüng steht mit ihrem Charakter der Wirklichkeit vielmehr am Ende alles Weges der Wissenschaftsbemühung, die anhebt und in allen wie immer verschiedenen Bestimmungèn geleitet ist von der Analysis des Ũnendlichkleinen; um dieser Kraft willen, dem Problem der Empfindungswirklichkeit, dem Problem des Daseins inbaltliche Bešstimmtheit, d. h. das Sein zu erżeugen, ebendarum wird das Infinitesimale Realität genannt. Das Infinitesimale ist die Instanż des Seins, der Realität für das Problem des Daseins, der Wirklichkeit. Realität besagt also das Mittel der Erzeugung der Gegenständlichkeit; die "Wirklichkeit" der Empfindung ist der unendlich ferne Problemstand, die Richtungskonstante solcher unendlichen Wissenschaftsarbeit. "Gegenstand der Natur" heisst also unendliche Vergegenständlichung durch naturwissenschaftliche Arbeit; Wirklichkeit, Dasein ist nur İdee und Aufgabe totaler Bestimming durch die methodisch reinen Mittel der Wissenschaft; Sein $=$ Realität $=$ Infinitesimales ist dieses methodisch reine Mittel, Dasein $=$ Wirklichkeit zu erzeugen und zu rechtfertigen aus der Homogeneität des wissenschaftlichen Denkens. Das Infinitesimale ist, weil es das Urmittel der Erkenntnis ist, Realität = Sein. Sein ist also gleich Erkenntnis, Erkenntnis gleich Sein; und darum ist Seín nicht'Dasein; sondern das Sein für das Dasein, der 
Hermann Cohens systematische Arbeit i. Dienste d. krit. Idealismus. 233

ursprungsreine Inhalt der Erkenntnis für das Problem der Erkenntnis.

Noch eine andere Bestimmung, die Cohen für das Infinitesimale aufnimmt, soll hervorgehoben werden, weil sich auch an sie leicht ein Missverhältnis anknüpft; aber auch deshalb, weil sie wie die eben erörterte die methodische Gesamtstimmung Cohens charakterisiert.

Die Methode des Infinitesimalen als das Urteil der Realität ist das erste der Urteile der Mathematik. Und also stehen wir im Gebiete der Zahl. Die Zahl ist mit dem Geruche des Subjektivismus behaftet; man "zählt" eben die Dinge; was geht das die Dinge an? So die Worte des Aristoteles. Dieser Verdacht des "Subjektivismus" verstärkt sich durch die Einsicht, dass die Einheit, mit der man eine Zahlenmannigfaltigkeit durchzählt, willkürlich ist; wird eine Bestimmung geändert, so kann dadurch eine Einheit in eine Mehrheit neuer Einheiten zerfällt werden. Also ist die Einheit immer relativisch zu einer Mehrheit bestimmt. Das heisst: die Einheit, mit der man zählt, ist nicht an sich Einheit, ist nicht absolut.

Ganz anders bezüglich der Infinitesimalzahl. Sie ist nicht bestimmt aus der Rücksicht auf eine Mehrheit; das dx ist nicht Zähleinheit, Mehrheitseinheit, sondern diejenige "Einheit", aus der das Endliche im Sinne des Integrals seinen Ursprung nimmt. Somit scheidet sich die "Einheit" des $d x$ scharf von der Zähl- und Mehrheitseinheit. Das Infinitesimale ist als Ursprung aller Endlichkeit und also anch aller relativierten Mehrheitseinheiten Absolutheit. Absolut heisst nicht "isoliert" oder "konstant": A'ber das Infinitesimale ist eben das letzte oder vielmehr das erste Element, mit dem die Wissenschaft Gegenständlichkeit beglaubigt, und also nicht erst durch eine Mehrheit legitimiert, die schon irgend wo und wie da wäre. So ist das Infinitesimale "das Sein", ursprüngliches Sein, sofern es Erkenntnis ist; und als schlechthin ursprüngliches Sein nicht relativiert auf eine Mannigfaltigkeit, die es als "Einheit", als ihre Einheit erst definierte, sondern es ist für die Integration des Endlichen ein letzthin Erstes, methodische Absolutheit.

Die methodische Kraft und systematische Konsequenz, die in der "Logik der reinen Erkenntnis" waltet, zeigt sich auch in dem Nacheinander der drei Urteilsarten der Mathematik. Das Urteíl der Realität ist das erste. Kant setzte als erste Kategorie 
die der Einheit, als zweite die der Vielheit. Aber es kann eine Einheit nur im Zusammenhange mit ihrer Mehrheit bestimmt werden and umgekehrt, wenn sie nicht in den schiefen Sinn der Einzelheit oder gar der Einzigkeit geraten will, welche beide der Tendenz der Zahl entsagen.

Und also darf es kein Urteil der Einheit geben, das ein Urteil der Mehrheit getrennt von sich hätte; und umgekehrt. Somit zieht Cohen in bedeutsamer Korrektur Kants die Kategorie der Einheit und die der Vielheit in das Eine Urteil der Mehrheit zusammen.

Es ist nötig, dieses Urteil der Ṃehrheit zu betrachten. Gemäss der fundamentalen Geltung des Denkgesetzzes des Ursprungs, dass das Denken der Wissenschaft seinen Inhalt in methodischer Kontinuität erzengen muss, kann weder die "Einheit", aus deren Wiederholung die Mehrheit entsteht, "gegeben" sein, noch das Mannigfaltige, über das die Einheit hingleitet und es zur Mehrheit artikuliert. Cohen kontrolliert sich für dieses Urteil am mathematischen Begriff der Reihe. Die Reihe ist ihrem allgemeinen Begriffe nach die Methode des Reikeens; und also liegt im Begriff der Reihe die Prospektive. Dieser Sinn kommt im Indefiniten der Reiheñunendlichkeit zụn Ausdruck. Nicht auf einen Anfang noch auf ein Ende ist bezogen, sondern das wird gefordert, dass es stets einen näehsten Schritt gẹbe. D̈iese Möglichkeit eines nächsten Sçbrittes wird im Begriff der Reihe als des Reihens, d. h. des Indefiniten, vorweggenommen. Dies Denken einer Antizípation des Folgenden ist der primäre Sinn der Indefiniten der Reihe. Und diese Antizipation ist niehts anderes als das Charakteristikum der 'Zeit. Zuerst die Zukunft, von der sich die Vergangenheit abhebt; angesichts des Noch-Nicht taucht das Nicht-Mehr auf. So entsteht die erste Form dër Mehrheit: in der Ersonderung der Vergangenheit von der ursprünglichen Tat der Zukunft. Als Organ vorzugsweise der Zukunft wird die Zeit somit zur Kategorie der Antizipation innerhalb des Urteils der Mehrheit.

Burch diese Formulierungen ist ein kräftiger Schritt weiter in der Erfüllung der Forderung getan, dass das Denken als Deñken der Wissenschät seinen Inhalt $\mathrm{zu}$ rechtfertigen und also zu erżeugen hat. Wird die Zeit zum Motiv des nächsten Schrittes, des nächsten Elementes, zum Organ der Vorwegnahme der Zukunft vielmehr denn zur retrospektiven Ordnung eines Nacheinander, so 
ist damit eine Auffassung der Zeit beseitigt, in der sie nur das Instrument, die „Form" ist, Vorstellungen in Reih' und Glied zu stellen, die aber schon anderweitig "gegeben" sind. Damit ist denn auch eine unlautere Methodik vermieden, die eklektisch haltlos zwischen Psychologie und Rationalismus hin und her schwankt.

Das soll uns nicht weiter beschäftigen, dass hiermit die Zahleinheit, die nur in der Zahlmehrheit zu denken ist, noch nicht vollständig bestimmt wurde; hier haben noch weitere Bestimmungen einzugreifen. Wir wollen uns nur der Frage entgegenstellen, ob es möglich und notwendig ist, die Zeit aus ihrer Kantischen Charakteristik als Anschauung herauszuheben und wieder mit dem aristotelischen Namen einer Kategorie zu kennzeichnen.

Man sollte meinen, dass für die grosse methodische und systematische Schwierigkeit, die in der Verbindung von Denken und Anschauung bei Kant liegt, der Blick nicht so schwer zu wecken ist. Man behauptet, es sei unmöglich, Zeit und Raum systematisch einheitlich unter das "Denken" zu stellen. Damit weist man ja aber selbst auf die methodische Schwierigkeit hin, wie "Anschauung“ und „Denken" hernach in die Einheit der Erkenntnis sollen zusammentreten können. Der „Anschauung“ wird das Vertrauen entgegengebracht, sie berge im Bathos der Erfahrung sicher vor den Verlockungen eines ungezügelten "Denkens"; das "Denken" aber und sein Begriff wird entleert, um ihm hernach aus der "Anschauung“ Gegenständlichkeit zufliessen zu lassen.

Das alles sind überaus gefährliche Formulierungen, nicht nur, weil dadurch Tür und Tor für jegliche Anmassung der Empfindung geöffnet ist, sondern vor allem: weil damit dạs Verständnis für das durchaus fundamentale Mittel naturwissenschaftlicher Erkenntnis, für die Infinitesimalrechnung ein für allemal verbant ist. Ist dies erkannt, so bleibt schlechthin keine andere Wahl, als den Erkenntnismitteln, die wir Zeit, Raum und Bewegung nennen, ganz innerhalb desjenigen systematischen Aufbaues, der von dem Charakter des infinitesimalen Denkens geleitet ist, einen Platz zu bestimmen, selbstverständlich unbeschadet ihrer vollen Eigenart. Ist diese letztere Reserve zngestanden (und wie sollte und müsste sie es nicht), so bedentet es einen Streit um Worte, will man diese drei Erkenntnisweisen nach wie vor als "Anschauung" im Gegensatze gegen das "Denken" festhalten; ein Streit um Worte, der die Sache aufs Spiel setzt, weil er den Blick für die Sache verfälscht. 
Es kann nicht als unsere Aufgabe betrachtet werden, allen Wandlungen im kritischen Apparate durch die "Logik der reinen Erkenntnis" nachzngehen. Wir wählten die Zeit aus, um an ihr zu zeigen, wie bestimmend das neue Fundament ist, das Cohen der Logik im Urteil des Ursprungs und im Urteil der Realität gegeben hat. Und wir taten es, weil bei Kant die Zeit allen Mitteln der Erkenntnis voraufgeht und durch alle Bestimmungen des Kantischen Systems hindurchwirkt: als Ort des Mannigfaltigen der Sinnlichkeit, als Grundlage des Schematismus der reinen Verstandesbegriffe, als entscheidende Waffe gegen die Amphibolie der Reflexionsbegriffe, als Platzbereiter für den Grenz- und Aufgabenbegriff des "Ding an sich", d. h. für die Ideen. Somit steht die Zeit bei Kant methodisch in Analogie zum Denkgesetż des Ursprungs und seiner Verwandlung innerbalb der Mathematik zum Urteil der Realität beí Cohen. Alle Befugnisse und Verantwortungen der Zeit bei Kant werden nunmehr auferlegt dem. Urteil des Ursprungs und seinen Dependenzen, zu denen die Zeit gehört, die aber jetzt als Dependenz der "Realität" unter der einheitlichen Methodik des Denkens als des Denkens der máthematischen Naturwissenschaft stẹt.

Die systematische Kraft, die vom Urteil des Ursprungs ausgeht, erschöpft sich nicht im Aufbau der „Logik der reinen ErKenñtñis"; man möchte sagen, dass sie sich steigert beim Übergạng von der "Logik" zum. Aufbau der "Ëthik des reinen Willens" (1904). Muss doch auch die Methode des Idealismus, die eine Philosophie als Wissenschaft zu instaurieren behauptet, auf dem Giebiete der Ethik ihre eigentliche Probe ablegen. Denn diese ist durch die Geschichte der Philosophie hindurch bis auf unsere Tage der Tummelplatz aller der buintscheckigen Kämpfer um die sogenannte Willensfreiheit und aller der Scharen, die von jenen her ihre Schlachtrufe erhalten.

Der Streit um die Willensfreiheit ist ein Streit um das Verhältnîs von Natur und Sittlichkeit; oder besser: um das Verhältnis der Gesetzlichkeit der Natur und derjenigen des Willens. Nun ist die Gesetzlichkeit der Natur methodisch deponiert in der unendlichen Arbeit der Naturwissenschaft, für die sich die Logik im Sinne einer Wissenschaft ihrer Prinzipien konstituiert; und entsprechend für die Prinzipien der Sittlichkeit: die Ethik. Somit 
handelt es sich bei dem Problem der Willensfreiheit methodisch und systematisch um das Verhältnis von Logik und Ethik.

Der Gedanke eines 'Verhältnisses von Logik und Ethik entspringt eigentlich erst durch Kants Unterscheidung des Seins vom Sollen. Damit ist das Gebiet der Ethik als Sportplatz für Naturalisten und Spiritualisten gesperrt worden. Es galt nicht mehr die Spielregel, den Willen hin und her zu zerren zwischen dem materiellen Daseinsgebiete und einem immateriellen; es war nicht mehr ein diskutables Problem, die Freiheit des Willens aus der Geschichte beweisen oder widerlegen $\mathrm{zu}$ wollen. Dem Tatsachengesetz der Natur wurde die Aufgabenidee des Sittlichen übergeordnet. Die Einsicht in die methodische Grenze der Kausalität wurde geschaffen und der Blick eröffnet für die methodische Eigenart des ethischen Problems.

Gleichwohl, wie weltgeschichtlich bedeutsam diese Tat Kants bleibt, den Streit um die Ethik aus einem Streit über die Disparatheit oder Uniformität zweier Dảseinsweisen in die Diskussion über eine methodische Unteršcheidung hinübergeleitet $\mathrm{zu}$ haben, gleichwohl bleibt diese Unterscheidung des Seins vom Sollen nicht völlig einwandfrei. Es entwíckeln sich aus dieser Unterscheidung zwei Bestimmungen der Ethik, deren eine den Anspruch der Logik überspannt, die andere den der Ethik.

Ist das Sollen nicht ein Sein, welchen Geltungswert hat dann das Gesetz des Willens? Wie kann es sich gegen die Gesetze der Natur behaupten? Ist das Sein die Objektivität des Geschehens, bleibt dann für das Sollen nicht nur das dunkle Gebiet des Subjektiviŝ̀mus übrig? Wie ist in diesen abgründlichen Gebieten der "Gesinnung", des "Gewissens" oder wie sonst noch sittliche Triebkräfte beissen mögen, ein Gesetz für Menschen in ihren realen Nöten zu begründen? Das ist die Schwierigkeit für die ethische Spekulation unserer Zeit, die ihre sittliche Welt sichern will. Mit dieser Unterscheidung des Seins und des Sollens bleibt der Begriff des Idealismus schwankend, seiner Durchschlagskraft beraubt; es muss die Stimmung fehlen, ihn als Realismus zu verstehen.

Das Sollen muss wieder als ein Sein begründet werden; aber, von der Geschichte belehrt, nach und vermöge einer methodischen Reinigung des Seins-Begriffs. Die ${ }_{n}$ Logik der reinen Erkenntnis" schūtzt uns nunmehr davor, das "Sein" als das „Dasein" verstehen zu müssen. 
Aber in der Unterscheidung von Sein und Wollen steckt zugleich die entgegengesetzte Tendenz: das Sittliche bedeutet gegenüber der Natur den Primat der praktischen Vernunft. Diesen Gedanken steigert Fichte zu der Entartung, dass aller Wahrheitswert der Seins-Erkenntnis nur als ein ethisches Postulat behauptbar sei, weil von dem Reiche sittlicher Wesen benötigt für die Zwecke dieser Welt. Damit verfällt das Sein der „Erscheinungswelt" in methodische Ab̉hängigkeit von der Sollenssicherheit der sittlichen Welt.

Vor beiden Entartungen bewahrt Cohen die Ethik, weil er den Unterșchied von Sein und Sollen im Sinne eines Gradunterschiedes der Geltung aufhebt. Das Sollen muss als Sein verstanden werden, als das ethische Sein mit anderen Bestimmungen als das logische Sein, aber in systematischem Zusammenhange, in methodischer Weiterführung des logischen Seins.

Der alte Gegensatz zwischen dem Sein der Natur, das in dèr Logik den Ort seiner Prinzipien zu erhalten hat, und demjènigen Geltüngsañspruch, der sịch das Sollen nennt, war ehedem formulierbar als Gegensat $\bar{z}$ "zwischen einem fremden Gegebenen und der eignen Wollung. Nūn lèrnten wir den Ausgang Cohens vom Denkgesetze des Ursprungss kennen, das allem İṇhalt der Erkenntnis als Forderung voranstand, die Gegenständlichkeit der Nàtur in unabgerissener Vertiefung von Prinzip rückwärts zu tieferem Prinzip zu begründen, oder vielmehr: heraufzuholen, zu ergründen; Gewinnung âlles Iñhalts des Denkens aùs der Tat des Denkens selbst, in unbeschränkter Selbstverwandlung der prinzipiellen Motive. Diéses Denkgesetz erhält seine erste Wandlung auf den Inhalt hin im mathematischen Gesetz des Infinitesimalen, im Urteil der Realität; Denken erzeugt sich als Sein, Sein ist Ursprïnglichkeit des̃ Denkens; das Sein ist in methodischer Reinheịt gedacht, befreit von dem methodischen Widerspruch des Unbegriffs einer fremden Gegebenheit.

Ist bei solcher Vepanlagung der Logik Gegensatz oder Harmonie zur Ethik noch ein Objekt des Streits? Ist Sein und Sollen vor allem andern im Lichte einer Unterscheidung zu sehen? Ist nicht vielmehr bei solcher Gestaltung der, Logik aus.der Kontrolle am Infinitesimalbegriffe in dieser ein Vorbau, ein Unterbau für die Ethik geschaffen?

In der „Ethik des reinen Wollens", die eine „Logik der reinen Erkenntnis" sich vorausgestellt weiss, tritt an Stelle des 
Gegensatzes oder der Unterscheidung als Grundgesetz an den Anfang das Gesetz, dass ein Zusammenhang und Einklang des theoretischen und ethischen Problemes bestehe.

Die Ethik ist durch die Logik bedingt. So spricht die Lauterkeit des sittlichen Bewusstseins, von dem sich die Geschichte durch allen Begeisterungsüberschwang für die eigene Aufgabe des Willens nicht abbringen liess. Es ist die Beziehung der Sittlichkeit zum Sein der Natur unzerrissen und straff zu erhalten; in dieser Tat besteht das, was wir Wahrheit nennen. Nicht die Ethik an sich hat Wahrheit; denn ihre "Idealität" schwebt in der Luft oder entweicht in die Leere einer Transzendenz. Nur der Zu'sammenhang beider, der Einklang beider Gebiete ist Wahrheit; denn er ist Bestand der Logik vor der Ethik, der Ethik vor der Logik.

Somit ist jenes obige Gesetz das Grundgesetz der Wahrheit. Dieses Grundgesetz der Wahrheit bestimmt allen Aufbau der Ethik, muss jeder Ethik voraufstehen. Denn dieses Grundgesetz bedeutet, dass das Denkgesetz des Ursprungs sich fortsetze durch die Gesamtheit aller Gebiete, die der wissenschaftlichen Erfassung zugänglich sind; dass es das Gesetz der Möglichkeit eines Systems der Philosophie sei. Das Grundgesetz der Wahrheit ist die Proklamation, dass die Methode der Logik sich in das nene Gebiet des Willens hinein fortzusetzen habe, dass auch dieses aus reinen Grundlegungen sich erbaue, gewiss in spezifischer Art, aber nur in kontinuierlicher Verwandlung des logischen Fundamentes. In dem Grundgesetz der Wahrheit wird der Lehrbegriff des Idealismus, dem în der Logik sein Fundament geschaffen ist, auf die Systemstufe der Ethik weiter geführt.

Steht das Grundgesetz der Wahrheit zur Disposition des Aufbaues der Ethik voran, so mag der Gedanke auftauchen, dass hier ein ethischer Intellektualismus eingeführt werden solle, dass der Wille seine Selbständ̆igkeit an das Denken preisgeben werde. Der klassische Idealismus weiss, dass das ethische Problem seine Selbständigkeit gegen das Denken und die Vorstellung zu schützen hat; aber zugleich und nicht minder gegen den Trieb und die Begehrung. Somit gilty:es erstens zu erkennen, dass es ein schäd-· liches Vorurteil ist, das Sittliche in der Gesinnung za gründen. Durch die Gesinnung wird der Wille zum blossen Gedanken abgeschwächt und seine Kollision mit der Religion bewirkt; vielmehr möchte die echte Gesinnung diejenige sein, die als notwendig zum 
Willeu die Tat, die Äusserung des Willens in der Handlung mit hinzurechnet. Aber zweitens gilt auch zu erkennen, dass nicht der Trieb und die Begehrung den Willen begründet. Denn der Trieb ist auf einen Gegenstand bezogen, der ein äusserer ist und also. nicht ein Willensobjekt sein kann. Wille muss reiner Wille sein, wie das Denken kraft des Denkgesetzes des Ursprungs reines Denken war. Für diesen Charakter des Willens setzt Cohen den Terminus "Tendenz", die das Analogon der (Iogischen) Realităt ist, sofern sie der Ursprung und also das Reine des "Affektes" ist. Der "Affekt" soll die Bedingung der Selbstbewegung erfüllen; das spricht sich' im Terminüs "Tendenz" aus. Wille ist Bewegung; und dass die Bewegung reine Bewegung sei, das besagt die Tendenz. Weil der Wille sich seinen gesamten Inbalt selbst erst erzeugen muss, so tritt aber zugleich auch sein $\mathrm{Zu}$ sammenhang mit der Logik zu Tage. Der Wille ist theoretisch zu begründen durch den Vorsatž, der den Sinn der "Aufgabe" zum Ausdruck bringt: In der Verbindung und Vereinigung des Affektes mit dem Denken entsteht die Handlung. In der Hand= lung erst vollzieht und vollendet sich das reine Wollen.

Wie unterscheidet sich nun aber der Inhalt des Willens von dem deș Denkens? Das Denken geht immer auf deñ Gegenstand, der als ein "äusserer" wie ein gegebener hingestellt werden muss. Im Willen handelt es sich um ein Inneres, das ein solches auch in der Tendenz und der Aufgabe bleiben soll. Dieses "Innere" bestimmt sich genauer als das $\mathrm{Subjekt}$ des Willens. Das $\mathrm{Be}$ wusstsein muș es sein, das sich in der Handlung entfaltet. Dieses müss sich in allêr Mannigfaltigkeit als Aufgabe durchsetzen; somit wird das "Subjekt" des Willens zum Selbstbewusst́sein.

Damit stehen wir vor dem Bedeutendsten der „Ethik des reịnen Willens".

In der Handlung erkennen wir die, Vereinigung von Affekt und Denken. Durch die Handlung wird das Innere als ein Äusseres dargestellt, obne es als ein Inneres aufzugeben. Nun ist Handlung nicht ein Bewegungsvorgang, der in einem Gegenstande verliefe. Handlung ist auch nicht Tat oder Triebbewegung, diè das Ding als ihr Bewegungsmotiv sich'zur Sache machte. Zur Handlung gehören zwei Subjekte. Das „Selbstbewusstsein" kann für den Willen und für die Handlung nicht „Berrusstsein des Selbst“ im Sinne des Einzigen bedeuten. Das Selbstbewusstsein und also die Handlung ist in erster Linie bedingt 
durch das Bewusstsein des Andern. Wer, ja: was ist „der Andere" ?

Ist der Begriff der Handlung nicht zu entwickeln ohne den Begriff des Andern, so ist erstens das "Ich" nicht eine Tatsache, sondern zu seinem Ursprung in genauer Kontinuität auf den "Andern" bezogen. Es könnte das Ich nicht definiert, nicht aus seinem Ursprung ergründet werden, wenn es nicht bedingt wäre durch den "Andern". Weil aber zweitens der Begriff der Handlung und also des Selbstbewusstseins nicht zu definieren ist ohne den Begriff des Andern, so bildet dieser eine Bedingung der. Möglichkeit der Handlung und des Selbstbewusstseins, das heisst eine reine Voraussetzung, eine reine Grundlegung. Das Selbstbew usstsein entspringt somit in der Korrelation des Andern und des Selbst. Im Begriff des Selbstbẹusstsein ist eine Mehrheit der Subjekte eine apriori-Voraussetzung.

Diese Konstruktion beruht auf dem Begriff der Handlung, für die sich der kritische Idealismus an der Wissenschaft des Rechts kontrolliert. In dem Rechtsbegriff der Handlung konstituiert sich das Selbstbewusstsein als das Bewusstsein des Selbst und des Andern, des Andern und darum des Selbst. Das àndere Subjekt ist also gar kein neues Problem; es ist von Anfang an in der Handlung mitgesetzt.

Bleiben wir hier erst einmal stehen. Es ist ohne weiteres klar, wie damit die Psychologie für alle Begründung der Ethik ausgeschlossen ist. Der Begriff der Handlung ist nicht aus dem Individuum abzuleiten; denn die Hypothesis, die reine Grundlegung "des Andern" ist ihre Vorbedingung. Aber zugleich damit wird ${ }_{n}$ der Andere" nicht durch den Affekt, den Affekt der Liebe erst herbeigezogen, dem Selbst in die Nähe gerückt als „der Nächste"; sondern das Selbst wird zu einem Inhalt, zu einem Innern erst durch das Äussere dieses Innern: den Nebenmenschen. Damit ist auch alle religiöse Begründung aus der Ethik ausgeschlossen. Der Andere wird vom Recht gefordert, und also von den realen Mächten des wissenschaftlichen Denkens.

Der reine Wille gibt sich in der Rechtswissenschaft und der Staatslehre mit wissenschaftlicher Genanigkeit und Unzweideutigkeit kund. Was die mathematische Naturwissenschaft für die Logik, das sinḍ sie für die Ethik; sie möchten geradezu als Mathematik der Geisteswissenschaften zu bezeichnen sein. So- 
dass sich die Ethik als Rechtsphilosophie durchführen muss, wie die Logik als Philosophie der mathematischen Naturwissenschaft.

Mit dieser Orientierung ist eine Korrektur an Kant von allergrösster Bedeutung vorgenommen. Kant batte die Anwendung der transzendentalen Methode im Gebiete der Ethik fallen lassen, denn er hatte die Ethik nicht am vorausstehenden Faktum der Rechtswissenschaft vollzogen, wie die Logik an dem der Naturwissenschaft. So stand die Rechtslehre ohne ihr prinzipielles Fundament da, das sie in den Grundlegungen der Ethik zu erfahren hat. Und die Tugendlehre büsste die nüchterne Realität des Denkens ein, die das Gebiet des Rechtes und des Staates durchwirkt, und musste in die Gebiete der Gesinnung und des religiösen Pathos zurückggehèn.

Durch die Orientierung an der Rechts- und Staatsleḥre erhält das Subjekt, auf das es in den Geisteswissenschaften ankommt, seinen Kontrollbegriff in der "juristischen Person“. In diesem Begriff liegt latent der Gegensatz gegen die einzelne Persoñ und liegt der Bézug auf die Genossenschaft. Die Genossenschaft lèhrt als das entschèidend Bedeutsame, dass es sich in ihr nicht um den Willen eines Einželnen hândelt und daŝs gleichwohl dieser Wille mehrerer Personen nicht äls ein gespaltener Wille 'gilt; sondern dasss in -ihm und núr in ihm die echte Einheit des Willens und demgemäss der Begriff des Rechtssubjektes zu seiner exakten Geltung gelangt. Der Gesamtwille tilgt die Einzeḷwillen, setzt sich an déren Stelle. Ünd nichts deštoweniger íst dieser repräsentative, dieser ideale Wille der eigentlich reäle. Denn von seinem Beschlusse hängt der Wille ùnd somit das Rechtssubjekt der Genossenschaft ab. Dieser geeinte, repräsentative, ideale Wille bildet die Einheit des Willens und die Einheit der Person.

$\because$. Dièse rechtswissensèhaftliche Darstellung und Begründung der idealen Person im Rechtssubjekte ist lehrreicher, überzeugender, weil präziser und prägnanter als alle sonstigen Erweiterungen des Selbștgefühls, mit denen man das Individuum von den Schranken des Eigensinns und der Selbstsucht, zu befreien liebt. Bei der juristischẹn Person genügt es nicht, dass die Eínzelnen sich ihrer Einzelheit entäussern; damit würde noch nicht das wahrhaft einheitliche Selbstbewusstsein zustandekommen, das in allen Einzelheiten bedingt als Rechtssubjekt in der Handlung sich vollzieht. Nicht um eine Erweiterung also des Selbstbewusstseins handelt es sich, wenn der Anmássung des Individuums gegenüber das Recht 
Hermann Cohens systematische Arbeit i. Dienste d. krit. Idealismus. .243

der Gemeinschaft auftaucht. Sondern gemäsis dem Rechtsbegriffe der Genossenschaft und der juristischen Person muss erst das Selbstbewusstsein definiert werden. Das ist der gewaltige methodische Gewinn, der aus dem Problem der juristischen Person der Genossenschaft zu ziehen ist, dass das Rechtsinstitut und der rechtswissenschaftliche Begriff den ethischen Begriff des Selbstbewusstseins des reinen Willens rechtfertigt und realisiert.

Auch die Ethik also ist vion nun an auf das Faktuin einer Wissenschaft bezogen, bei dem sich die transzendentale Frage erhebt, wie sie möglich sei. Dieser Fortschritt des kritischen Idealismus wird. der Geschichte der Philosophie nicht verloren gehen.

Es soll von uns unerörtert bleiben, warum Cohen die Soziologie als Wissenschaftsvorausbestand für eine Ethik abweist. Es möge nur ausgesprochen werden, dass sie abgelehnt wird, weil sie die Gemeiṇschaft als eine geschichtliche Relativität dẹr Entwicklung darstellt. Die Pädagogik wird nicht unter die $\mathrm{Be}$ trachtung gestellt, ein Wissenschaftsvorausbestand für Ethilk zu sein. Ist sie es, so müsste die Ethik alsdann über die spezifische Methode des Aufbaues einer "Wissenschaft von der Erziehung" die transzendentale Frage nach ihrer Möglichkeit erbeben. Die Erörterung über Recht oder Unrecht dieser Beschränkung Cohens auf die Rechts- und Staatslehre wird die Geschichte zu vollziehen haben. Aber stets wird solches zu geschehen haben unter dem fundamentalen Leitgedanken Cohens, dass es gilt, auch die Ethik vor dem Verfall in eigenmächtig spekulierende Konkurrenz mit. den Einzelwissenschaften zu schützen; auch auf ethischer Stufe geht Philosophie den Weg der Wissenschaft allein durch den $\mathrm{Be}$ zug auf die konstruktiven Einzelwissenschaften vom Willen und der Handlung, wie umgekehrt diese ihres Fundamentes nur durch die Kritik der Ethik sicher werden.

Gehen wir noch einmal an den wichtigen Begriff der Genossenschaft hinan. Ist nicht der Gedanke nabeliegend; dass die Genossenschaften durch Einzelzwecke bestimmt und begrenzt sind, dass der Begriff aus ökonomischen Bedürfnissen entstanden ist? Es fehlt in unserer Darstellung des Begriffs der Genossenschaft, dieses Musterbildes für das Selbstbewusstsein, noch eine Auszeichnang derjenigen Gestaltung, die der Forderung der Einheit des 
Willens durch die Allheit ihrer Glieder und ihrer Willen genügt. Die Genossenschaft gelangt zu ideeller Reinheit in der Idee des Staates.

Wir haben uns zunächst davon zu befreien, im Staat nur die reale Institution einer Exekutive zu sehen und also ihn mit Regierung zu verwechseln. Aber andererseits besteht nicht die -Absicht, einen fiktiven Begriff, die Fiktion eines Staatsideals einzuführen. So ist denn der Begriff des Staats als Aufgabenbegriff zu bewerten. Als Begriff der Aufgabe der Allheit des Willenssubjektes und dadurch der ethische Leitbegriff des Selbstbewusstseins stellt er die Idee einer Verfassung sittlicher Subjekte dar. Die Staatseinheit hängt davon $a b$, dass jedes Wesen, das, weil sittliches, weil rechtliches Subjekt, des reinen Willens fähig ist, in diese Idee des Staates einbezogen seí; und umgekehrt ist von der Anteilnahme des Einzelnen an dem Aufgabenbegriff des Staates der Vollzug seines Selbstbewusstseins abhängig. Der Staat, als Aufgabe, ist die Aufgabe des Selbstbewusstseins; und also die Einhëit von Subjekt und Objekt im Willen. Denn Einheit des Willens ist nichts als diese Allheit dér Staatsidee; und diese Einheit ist der Inhalt des Willèns, anf den er in allen seinen Handlungen sich zu beziehen hat. Nur in diesem Objekte kann der Einzelne sein Subjekt, sein Selbstbewusstsein als die unendliche Aufgabe seines reinen Willens erlangen und behaupten. Im Staatsbegriff wird somit das Ich zür reiństen Entfaltung $\mathrm{zu}$ bringen sein, indem der Andere zum: „Du" verwandelt wird. Unter Leitung der Staatsidee, des Allheitsbegriffs der juristischen Perșon kann ich es verstehen und beherzigen, dașs ich nicht in meinèr natürlichen Individualität das Selbstbewusstsein des Willens produzieren kann; und auch nicht dadurch, dass ich mich in Liebe und Enthusiasmus über die konzentrischen Kreise verschieden weiter und unterschiedlich verpflichtender Gemeinsc̀haften "werktätig " auszubreiten tràchte, sondern dadưrch allein, dass ich in derjenigen Bestimmtheit und Exaktheit, welche Recht und Gesetz allein ermöglicht, und gemäss derjenigen Allheit, -welche der Staatsgedanke allein als Einheit vollzieht, alles Selbstischen mich begebe und mein Ich nur in der Korrelation von Ich und $\mathrm{Du}$ denken und wollen lerne. $\mathrm{Zu}$ diesem Lernen gehört theoretische Kultur. Und indem der Staat mehr und besser, als Volk und Kirche dies tun, die theoretische Kultur in seine Befugnisse aufnimmt, wie dies die Aufgabe des Selbst- 
bewusstseins vorzugsweise fordert, so dient er dadurch auch der Liebe zu Volk und Vaterland.

Bis hierher erörterten wir die konstitutiven Begriffe der „Ethik des reinen Willens": den reinen Willen nebst der Handlung und das Selbstbewusstsein. Das Selbstbewusstsein vereinigt das Individuum und den Staat. Und der reine Wille, wie er zur Handlung wird, ist der Typus aller Betätigungsweisen des sittlichen Subjektes. Bei diesem Fundamente ist streng genommen der Mensch noch im Hintergrunde. Wichtiger und interessanter ist die juristische Person.

Aber das Interesse an ihr besteht doch darin, dass sie als Vorbild dienen kann und dienen soll für den Menschen und seine Individualität. Im letzten Ziele und Grunde muss doch die Ethik für den Menschen gelten.

Dieser Begriff des Menschen muss nun in den Vordergrand gerückt werden; und es geschieht, indem der Bëgriff der Freiheit zur Klarheit gebracht wird. Der Geist des Menschen ist seine Freiheit, und nur durch sie wird die Ethik die Lehre vom Menschen.

Der Begriff der Freiheit wurde in aller Geschichte als überaus schwieriger Begriff empfunden. Diese Sprödigkeit rührte daher, dass die Freiheit als eine Tatsache, als eine Kraft und Macht gedacht wurde, anstatt sie als eine Idee, als Aufgabe zu bestimmen. Ist Freiheit die Tatsache eines gewissen "Vermögens", wie entspringt dann die Handlung? Als absoluter Anfang, entsprungen der absoluten Person. So steht der Begriff der Freiheit da als Widerspruch zur Kausalität. Das ist nun die neue Wendung, die von Kant ausgeht: dass das Problem der Freiheit von der absoluten Person der alten Metaphysik übergeht auf eine Ursprünglichkeit des Gesetzes für die Handlung. Gibt es ein Gesetz, das die Handlung bestimnt und zugleich in methodischer Eigenart über der Kausalität selbständig ist, sodass der reine Wille die Handlung als einen Oberbau in methodisch absoluter Gesetzgebung, also in einer Selbstgesetzgebung anfängt? So kehrt sich die Frage ab von der Metaphysik absoluter Vermögen und. Kräfte hin auf die methodische Selbständigkeit der Gesetzgebung. So entspringt der neue Kantische Sinn der Freiheit: Es handelt sich nicht mehr um Eingriffe in das Bereich der Kausalität, sondern um die Autonomie aus der Methodologie von $\mathrm{Z}_{w e c k}$ und Mittel. Und nun fragt es sich: hat die Handlung einen absoluten Zweck, das heisst einen Zweck, für den der Mensch sich 
nicht nur als Mittel und Werkzeug aufreibt, sondern in welchem er, weil Mensch, Selbstzweck bleibt? Es geht jetzt also die Frage nach der Freiheit des Willens nicht auf eine erste Ursache, sondern auf einen letzten Zweck. Freiheit wird umgebildet in den Endzweck und Selbstzweck. Durch den Selbstzweck ist der Begriff der Person begründet worden.

Damit änderte sich der Sinn des Selbst. Es steht nicht als absoluter kausaler Anfang voraus $\dot{d} \dot{a}$, sondern am Ende aller Zwecke als der Endzweck und als Aufgabe. Das Selbst wird Idee und Aufgabe aus der Kraft der methodisch eignen Gesetzgebung, Das ist der Sinn der Autonomie.

Im Begriff der Autonomie müssen vier Bedeutungen auseinandergelegt werden.

Zunächst ist Autonomie also Selbstgesetzgebung. Als solche ist, sie der Widerspruch zur Heteronomie. Heteronomie ist Pathologie; und also die Selbstgesetzgebung der Widerspruch zur Selbsitsucht. Es ist das Vertrauen auf das reine Denken, worauf die Selbstgesetzgebung berubt, in dẹm Grundgesetze der Wahrheit hat sie ihre Wurzel. Denn die Selbstgesetzgebung muss dem Denkgesetze des Ursprungs entsprechen und ihren Inhalt in ursprungsreinen, methodisch lauteren Voraussetzungen begründen, e r.gründen. Dieser Inhalt der Selbstgesetzgebung ist das Selbst. Es ist in keiner Weise und in keiner noch so idealen Gestalt vorhèr vorhanden, bevor es sich darlegt. Denn die Selbstgesetzgebung ist nicht etwa die Gesetzgebung aus dem Selbst, sondern reàlisiert sich in unendlichen Schritten zum Selbst, als Selbst. Darüber liegt eine grosse UÚnklạrheit im Begriffe der Autonomie bei Kant, ăls wäre das Selbst schỏn gegeben und teile sich in den sittlichen Hàndlungen nur mit. Vielmehr erzeugt die Gesetzgebung auf ihrem unendlichen Wege das sittliche Selbst in seinem geschichtlichen Dasein, in der sittlichen Handlung.

Dass also das Selbst erstehe im Schritt vor Schritt der Gesetzgebung, drückt zugleich aus, dass es sich innerhalb jedes einzelñen Inhâltes der Handlung, der Handlungen realisiere. Jede einzelne Handlung muss mit jedem ihrer Schritte die Aufgabe des Selbst, die Realisierung des Selbst weiter führen. So stehen Handlung und Gesetzgebung zusammen. Jede Handlung ist ein Einżelakt der realisierenden Gesetzgebung, eine Einzelbestimmung zum Selbst, 
Darnach ist zweitens die Autonomie Selbstbestimmung. Sie hat nicht in der abstrakten Weite eines starr allgemeinen Gesetzes zu verbleiben, das vor den brennenden Einzelfragen des Lebens versagte. Das ist ein Vorwurf, den man fälschlicherweise der Antonomie bei Kant gemacht hat, weil man sich den Blick nicht festigen konnte für die methodische Allgewalt in jenem Wortè Kants, das àls ewiges Denkmal der - Kraft sittlichen Denkens bestehen bleiben wird: Handle so, dass du die Menschheit in dir wie in der Person eines jeden Andern niemals bloss als Mittel, sondern jederzeit zugleich als Zweck brauchst. Autonomie ist Selbstbestimmung, weil sie sich in den Einzelinhalt der Handlung vertieft, sich des Einzelinhalts bemächtigt; als Methode soll sie die Bestimmtheit der Wirklichkeit meistern. Und das Selbst soll in der Bestimmtheit des Einzelfalls der Handlung, der als ein Einzelfall innerhalb der allgemeinen Methodologie der Autonomie seine Bestimmtheit erfährt, zu eigner Bestimmung' und $\mathrm{Be}-$ stimmtheit gelangen.

Hieraus folgt, dass Autonomie dèn Sinn der'Selbstverantwortung habe; denn das Gesetz hat sich in der Einzelforderung der Handlung durch Selbstgesetzgebung durchgesetzt, und die Handlung als Selbstbestimmung vollzogen. Um aber die Wirklichkeit, die an sich und ausserhalb des Willens ein kausaler Vorgang ist, als Mittel in den Dienst des Willenszweckes zu stellen, muss der Vorgang zunächst als ein kausaler durchdacht sein. Die Klarheit des kausalen Denkens ist Voraussetzung der Beștimmung zur Handlung. Also betätigt sich die Selbstverantw̄ortung auf ihrem ganzen Wege hauptsächlich als kausales Denken. Keineswegs darf aber der Anteil des Affektes ausgeschaltet werden. Denn ohne ihn würde der Unterschied zwischen culpa und dolus verschwinden; und das Verbrechen zu einem blossen Denkfehler entsittlicht werden. Der Richter mag zu einem Freispruch kommen, sofern er sich seines Urteils über den Angeklagten enthält. Aber muss sich der Richter enthalten, dem Angeklagten die Tat zuzurechnen, so ist ihm doch keine Macht gegeben, den Angeklagten seiner Schuld ledig zu erklären. Für den Angeklagten ist die Selbstverantwortung damit nieht erledigt, dass das Recht seine Schranke hat. So ist die These aufzustellen, dass bezïglich des Richters die Zurechnung prinzipiell von der Schuldfrage abzutrennen sei. Die Selbstverantwortung hört niemals auf. Denn die Kultur hört auf, eine menschliche zu sein, wenn sie dem Spruche 
sich entziehen wollte, dass der Mensch in jeglicher Einzelbeit seiner Handlung ihr Urheber ist. Hier erkennen wir die Eigenart der Ethik gegenüber dem Recht: in der Bestimmung des sittlichen Selbstbewasstseins. So wird der Begriff der Selbstrerantwortang zur Selbsterkenntnis, der dritten Bedentung der Autonomie.

Zurechnung und Strafe sollen als juistische Obliegenheiten für den Richter von der ethischen Forderung der Selbsterkenntnis, der Schulderkenntnis getrennt sein; nicht aber für das Selbst. Vielmehr tritt die Strafe unter den Gesichtspunkt der Bessernng. Dies Prinzip der Besserung sichert ein Mass und eine Grenze der Strafe, die die Selbsterkenntnis zuerkennt und anerkennt. Denn die conditio sine qua non der Selbst-Besserung ist die Selbsterhaltung. Zurechnung und Strafe darf niemals die Grenzen der Selbsterhaltung überschreiten. Denn wird sie überschritten, so wird die unendliche Aufgabe und damit aller vollzogener endlicher Weg gemäss dieser Aufgabe preisgegeben. Somit wird das Prinzip der Selbstbesserung zum Prinzip eines Schutzes der Selbsterhaltung. Und auch dies ist eine strikte Folgerung aus dem Prinzip der Antonomie im Sinne der Selbsterhaltung, dass, wie es keinen guten Menschen gibt, so auch der Gedanke eines bösen Menschen nicht gefasst werden darf. Darnach möchte die Selbsterhaltung zu einem sichereren Leitbegriff der Ethik erwachsen sein, als er in Begtiff der "Würde“ sich anbietet. Das ist der vierte Sinn der Autonomie, dass sie Selbsterhaltung zn bedeuten habe.

In diesen Bedeatungen der Autonomie und der Freiheit sind die Merkmale im ethischen Problembegriffe des Menschen bestimmt, von deren Geltung die Realisierung deș Selbstbewusstseins abhängt. Diese Merkmale bédeuten ihrem höchsten Sinne nach nur Aufgaben. Somit ist denn zi fragen: Welche Bürgschaft gibt es, dass diese Aufgaben der Lösung fähig sind? Wie steht es um die "Wirklichkeit" des Sittlichen?

Zunächst ist das Grundgesetz der Wahrheit das sichere Fuñdament dieser Frage: es soll strengste theoretische Harmonie zwischen dem Reiche der Logik und der Ethik herrschen. Aber das genügt nicht.

Die Wirklichkeit der Natur ist auf die Grundlage des Raumes gestellt; während wir für das "Innere" des Willens die Zeit als das Motiv der Antiżipation, der Zukunft in Anspruch nehmen. Nun bedentet gemäss der "Logik der reinen Erkenntnis" der Raum den unendlichen Zusammenschluss, Allheit; die Zeit unab- 
geschlossene Vorwegnahme der Zukunft. Geht nun die Frage auf die Wirklichkeit des Sittlichen, so muss der Wille sich ans seinem Motiv der Zukunft ein Analogon der Raumunendlichkeit schaffen. Dieses Wirklichkeitsanalogon des Sittlichen ist die Ewigkeit. Ewigkeit bedeutet dem Willen nicht ewige Dauer; denn es gibt keinen Stillstand für ihn. Ewigkeit ist also nicht ein Zeitmesser, sondern sie ist rein ein ethischer Begriff. Der Wille schafft sich in der Ewigkeit die Bürgschaft seiner Wirklichkeit. Somit bedeutet Ewigkeit an sich nicht ewige Zeit, noch ewigen Ort, sondern ewige Arbeit des Willens.

Hieraus ergeben sich drei Momente der Betrachtung. Nicht, wie in der Natur ein einzelner Fall das Gesetz dieses Falles zur Darstellung bringt, nicht so findet der reine Wille in ingend einer einzelnen Handlung sein Dasein, seine Darstellung. Alle einzelne Handlung ist ein Unvollkommenes. Gleichwohl aber ist jede einzelne Handlung des reinen Willens nicht ein Widerspruch zum Gesetz dieses Willens, sondern wie eine Stufe und Ansatz zu höherem Schreiten aus demselben Geist, auf demselben Einen Wege der Vervollkommnung. Aber dass es eine Vervollkommnung, der Eine Weg einer Vervollkommnung ist, diese Gewissheit erhebt sich aus dem Ewigkeitsblick der Arbeit, dass der Weg der Arbeit sich eines unendlich fernen Zielpunktes sicher weiss. Dieser Ewigkeitsblick der Arbeit ist die Vollkommenheit der Arbeit.

Diese drei Momente schliessen sich zusammen zum sittlichen Ideal. Im Ideal schafft der Wille sich sein Sein.

Wie das Denken die Realität, das Sein der Natur ist, so ist nun das Wollen das ${ }^{9}$ Sein des Ideals. Aber das sittliche Ideal hat nicht die Wirklichkeit der Natur. Hier taucht dann wieder die Kantische Unterscheidung von Sein und Sollen auf. Das Einzelne jeder Handlung ist unvollkommen; aber die Ewigkeit der Vervollkommnung nimmt das Unvollkommene in sich auf, hält es in sich zusammen und macht es im Ganzen der ewigen Arbeit mit zur Wirklichkeit, die Ideal heisst, das Ideal, das in jedem Atemzuge unvollkommene Wirklichkeit wird. So offenbart sich im Ideal das neue Sein des Selbstbewusstseins.

In der Richtung auf den Staat soll es zu seiner Wirklichkeit gelangen. Soll es! Aber wann wird jener Staat in Wirklichkeit erscheinen, der Staat des Ideals? Muss für die Ewigkeit des sittlichen Ideals nicht zavor die Natur nach Zeit und Raum unendlich gesichert sein? Der Begriff, der dies zu leisten hat, 
darf also nicht lediglich in der Ethik seine Bedeutung erschöpfen, noch lediglich in der Logik als der Logik der Natur. Also genügt das sittliche Ideal selbst nicht, noch die Natur an sich. Es ist notwendig, dass die beiden Glieder des Systems 'zu einander in Verhältnis treten. Zwar hatte das Grundgesetz der Wabrheit gefordert, dass zwischen dem Problem der Ethik und der Logik ein Żusammenhang bestehen muss. Aber es muss die Gewissheit des Vollzuges dieses Zusammenhanges. bestehen; die erforderliche Verbindung beider Glieder muss ermöglicht werden. Denn von der Ewigkeit des sittlichen Fortschritts her, vom Ideale des sittlichen Seins erhebt sich die bange Frage, ob die Natur, die tote wie die lebendige, in solchen Entwickelungen ihrerselbst fortwirke, wie sie als negative Bedingung von -dem Gedanken der Ewigkeit géfordert werden. Es muss für die Harmonie der beiden Welten der Bègriff der Vorsehung beglaubigt werden. Die Ewigkeit des. Ideals nuuss gesichert sein durch die Vorsehung Gottes in der Natur, für die Sittlichkeit. Den Zusammenhang der ethischen Selbsterhaltung mit der Erhaltung der Natur, wie auch ihre Bewegungsformen sich wandeln mögen, diese Wahrheit nenñen wir Gott. Durch die Idee Gottes als Idee der Wahrheit w̌ird der Sieg des Guten vẹrbürgt, weil sich dem sittlichen Ideal die Perfektibilität der Entwivicklung auf Seiten der Natur nicht versagen wird. . Also garantiert es Gott soll es die Idee Gottes garantieren. Gott ist also Idee der Wahrheit;-das heisst: tiefstes Problem der sittlichen Erkenntnis; um meine sittliche Arbeit uñd die sittliche Notwendigkeit ihres Vollzuges handelt es sich bei diesem Problem nicht, die steht in ihrem Gesetze, in der Richtung ihres Weges und im Zukunftssinn ihrer Aufgabe fest. Die Sittlichkeit besteht in dem Inbegriff der ethischen Begriffe; da ist Gott nicht im Spiele. Und auch die Natur besteht im Inbegriffe ihrer logischén Gesetże; und aụch dabei ist Gott ausser Betracht. So bedeutet er also bezüglich beider Arten von Erkenntnissen eine Transzendenz. Aber diese Transzendenz Gottes ist eine Idee der Erkenntnis und also von methodischer, realisierender Bedeutung: dass nunmehr die Natur nicht transzendent bleibe der Sittlichkeit, noch die Sittlichkeit für die Natur. -

Wir können unsere Charakteristik des systematischen Werkes Hër̆mann Coheñs hiermit schliessen. Es zeigte sich, dass wie in der „Ethik des reinen Willens"., so auch in der „Logik der reinen 
Hermann Cohens systematische Arbeit i. Dienste d. krit. Idealismus. 251

Erkenntnis" ein terminologischer Apparat arbeitet, der mit dem Kantischen kaum noch Gemeinsames hat. Aber der methodische Geist ist in völliger Reinheit der streng kritische, der das Vermächtnis Kants in sich aufgenommen und zu grösserer Konsequenz hinaufgeführt hat.

Im März dieses Jahres folgte diesen beiden Werken des Cohenschen Systems der Philosophie das dritte: die zweibändige "Aesthetik des reinen Gefühls". Ein solches Buch erfordert eine längere Zeit als die Spanne eines Monats, damit man sich in seinem Problemgebäude so weit zuhause fühle, dass ein hinlänglich klarer Grundriss und Aufriss möglich ist.

So muss denn dieser Versuch, von Cohenscher Arbeit im Dienste des kritischen Idealismus ein Bild alfresco zu entwerfen, als Torso sich bekennen.

Abër dieses Eingeständnis geschieht fröhlichen Mutes. Denn es ist nicht eine bittere Notwendigkeit, die uns veranlasst, Stillstand und Umschau zu halten, sondern ein freundliches Gebot, den Feiertag zu heiligen; auf welchen Feiertag für uns und unsern Lehrer des kritischen Idealismus Werktage folgen werden voll Sonne und Segen. 